\title{
A GENERALIZATION OF D'ALEMBERT'S FUNCTIONAL EQUATION
}

\author{
STANLEY GUDDER
}

(Communicated by Andrew M. Bruckner)

\begin{abstract}
Recent investigations in the foundations of physics have shown that a quantum mechanical influence function satisfies a generalized d'Alembert functional equation. The present paper derives the solutions for this equation on a topological linear space.
\end{abstract}

\section{INTRODUCTION}

D'Alembert's equation is one of the most important functional equations $[1-4,7]$. This equation was originally introduced as a tool for studying the vibrating string problem and for axiomatic investigations of the parallelogram law for addition of force vectors $[5,6]$. It has been applied in noneuclidean mechanics [4] and harmonic analysis [10]. A function $u: \mathbb{R} \rightarrow \mathbb{R}$ is said to satisfy d'Alembert's equation if

$$
u(x+y)+u(x-y)=2 u(x) u(y)
$$

for all $x, y \in \mathbb{R}$. A classical result states that the only continuous solutions of (1.1) are $u(x) \equiv 0, u(x)=\cos a x$, and $u(x)=\cosh a x[2,4]$.

Recent investigations in the mathematical foundations of quantum mechanics have resulted in a generalization of (1.1). In his studies of discrete models for classical and quantum physics, Hemion introduced the concept of an influence $u: \mathbb{R} \rightarrow \mathbb{R}[8,9]$. The function $u$ provides a measure of the influence between physical states (or configurations). By applying the principle of strong causality (the future cannot influence the past), Hemion has argued that $u$ must satisfy the following causal condition

$$
\sum_{i=1}^{n} u\left(y_{i}\right)=0 \Rightarrow \sum_{i=1}^{n}\left[u\left(x+y_{i}\right)+u\left(x-y_{i}\right)\right]=0
$$

for all $x \in \mathbb{R}$. It is clear that (1.1) implies (1.2), so (1.2) provides an interesting generalization of d'Alembert's equation. A physical interpretation of (1.2) states that if a present total influence vanishes, then it still vanishes when future effects are included.

Received by the editors November 29, 1990.

1991 Mathematics Subject Classification. Primary 39B20, 39B70.

(C) 1992 American Mathematical Society $0002-9939 / 92 \$ 1.00+\$ .25$ per page 
We shall find it useful to introduce the following closely related condition:

$$
\begin{aligned}
\sum_{i=1}^{n} u\left(x_{i}\right) & =\sum_{i=1}^{m} u\left(y_{i}\right) \Rightarrow \sum_{i=1}^{n}\left[u\left(x+x_{i}\right)+u\left(x-x_{i}\right)\right] \\
& =\sum_{i=1}^{m}\left[u\left(x+y_{i}\right)+u\left(x-y_{i}\right)\right]
\end{aligned}
$$

for all $x \in \mathbb{R}$. This condition can be given a physical interpretation similar to that of (1.2). Of course (1.3) is also a generalization of (1.1). It will follow from our work that (1.2) and (1.3) are equivalent if $u$ is continuous and has a zero. If in addition, $u(0)=1$, then (1.1)-(1.3) are all equivalent.

Hemion has shown that if $u: \mathbb{R} \rightarrow \mathbb{R}$ is periodic with period $2 \pi$, even, continuous, monotone on $[0, \pi]$, satisfies a condition slightly stronger than (1.2), and $u(0)=-u(\pi)=1$, then $u(x)=\cos x$ for all $x \in \mathbb{R}$. In this paper, we present a vast generalization of Hemion's result. We cannot only dispense with many of Hemion's conditions, but our theorem applies to a real topological linear space $V$ and gives a classification of solutions that is similar to the classical result. Although the main application of our theorem is for the case $V=\mathbb{R}$, it may also be important when $V$ is a Hilbert space (Corollary 4) since quantum states are frequently represented by unit vectors in a Hilbert space.

\section{SOLUTIONS OF THE FUNCTIONAL EQUATION}

We now present our main result together with some corollaries. Let $V$ be a real topological linear space. A subset $A \subseteq V$ is balanced if $t A \subseteq A$ for $|t| \leq 1$ and absorbing if for every $x \in V$ there exists an $\varepsilon>0$ such that $t x \in A$ for $|t|<\varepsilon$. Recall that every neighborhood of 0 is absorbing and includes a balanced neighborhood of 0 [13]. We say that a map $u: V \rightarrow \mathbb{R}$ is $\mathrm{Cl}$ (C2) if $u$ satisfies (1.2) [(1.3)] for every $x \in V$.

Theorem 1. If $u: V \rightarrow \mathbb{R}$ is continuous and $\mathrm{C} 2$, then there exists a continuous linear functional $f: V \rightarrow \mathbb{R}$ such that either $u(x)=u(0) \cos f(x)$ for all $x \in V$ or $u(x)=u(0) \cosh f(x)$ for all $x \in V$. Moreover, $f$ is unique in the sense that if $u \neq 0$ and $u(x)=u(0) \cos g(x)$ or $u(x)=u(0) \cosh g(x)$ for a continuous linear functional $g$, then $g= \pm f$.

Proof. We may assume $u \neq 0$, since otherwise, we are finished. We may also assume $u \neq 1$, since otherwise, we let $f=0$ and again we are finished. If $u(0)=0$, then $u(0)+u(0)=u(0)$. Applying (1.3) gives $4 u(x)=2 u(x)$ for every $x \in V$. Hence, $u=0$, which is a contradiction, so $u(0) \neq 0$. We may assume that $u(0)=1$, since otherwise, we could consider $v=u / u(0)$ and $v$ satisfies the hypotheses of the theorem. We now consider two cases, either $u$ has a zero or does not.

Suppose $u$ has a zero so that there exists a $z \in V$ such that $u(z)=0$. Since $u(z)+u(z)=u(z)$, applying (1.3) gives

$$
u(x+z)+u(x-z)=0
$$

for all $x \in V$. We now show that $u$ is C1. If $\sum u\left(y_{i}\right)=0$, then $\sum u\left(y_{i}\right)=$ $u(z)$. Applying (1.3) and (2.1) gives

$$
\sum\left[u\left(x+y_{i}\right)+u\left(x-y_{i}\right)\right]=u(x+z)+u(x-z)=0
$$


for every $x \in V$. Hence, (1.2) holds so $u$ is C1. Letting $x=z$ in (2.1) gives $u(2 z)=-1$, and replacing $x$ by $x-z$ in (2.1) gives $u(x-2 z)=-u(x)$ for all $x \in V$. Assume that $u(y)$ is rational and $u(y)=s / t$ for integers $s, t$ with $t \neq 0$. Suppose $u(y)>0$, in which case we can assume that $s>0, t>0$. Let $n=s+t, y_{1}=\cdots=y_{s}=2 z, y_{s+1}=\cdots=y_{n}=y$. Then

$$
\sum_{i=1}^{n} u\left(y_{i}\right)=\sum_{i=1}^{s} u\left(y_{i}\right)+\sum_{i=s+1}^{n} u\left(y_{i}\right)=-s+t u(y)=0 .
$$

Applying (1.2) we have for every $x \in V$

$$
\begin{aligned}
0 & =\sum_{i=1}^{n}\left[u\left(x+y_{i}\right)+u\left(x-y_{i}\right)\right] \\
& =s[u(x+2 z)+u(x-2 z)]+t[u(x+y)+u(x-y)] \\
& =-2 s u(x)+t[u(x+y)+u(x-y)] .
\end{aligned}
$$

Hence,

$$
u(x+y)+u(x-y)=(2 s / t) u(x)=2 u(x) u(y) .
$$

Of course, this is d'Alembert's equation. If $u(y)=0$ then (2.2) follows directly from (1.2). Now suppose $u(y)<0$, in which case we can assume that $t>0$ and $s<0$. Let $n=|s|+t, y_{1}=\cdots=y_{|s|}=0, y_{|s|+1}=\cdots=y_{n}=y$. Then $\sum_{i=1}^{n} u\left(y_{i}\right)=|s|+t u(y)=0$. Applying (1.2) gives for every $x \in V$

$$
0=\sum_{i=1}^{n}\left[u\left(x+y_{i}\right)+u\left(x-y_{i}\right)\right]=2|s| u(x)+t[u(x+y)+u(x-y)] .
$$

Hence,

$$
u(x+y)+u(x-y)=-(2|s| / t) u(x)=2 u(x) u(y) .
$$

We conclude that (2.2) holds whenever $u(y)$ is rational. Letting $x=y$ in (2.2) gives

$$
1+u(2 y)=2[u(y)]^{2}
$$

whenever $u(y)$ is rational.

We now show that $u$ is not identically 1 on any neighborhood $N$ of 0 . If it were then applying (2.3) for $y \in N$ gives $u(2 y)=2[u(y)]^{2}-1=1$, so $u$ is identically 1 on $2 N$. Continuing, $u$ is identically 1 on $2^{n} N$ for every positive integer $n$. Since $N$ is absorbing, we conclude that $u=1$ on $V$, which is a contradiction. We now show that for any neighborhood $N$ of 0 there exists a $y \in N$ such that $u(y)$ is rational and $u(y) \neq 1$. Indeed, let $M \subseteq N$ be a balanced neighborhood of 0 . Since $u$ is not identically 1 on $M$, there exists an $x \in M$ such that $u(x) \neq 1$. Now the set $A=\{t x: 0 \leq t \leq 1\}$ is connected and $u \mid A$ is continuous. Hence, $u(A)$ is connected so $u$ attains every value between 1 and $u(x)$ (the intermediate value theorem). Hence, there is a $y \in A$ such that $u(y)$ is rational and $u(y) \neq 1$. We next show that $u$ cannot be identically a constant $c \neq 0$ on any neighborhood. Indeed, suppose $u(x)=c \neq 0$ for all $x \in N(w)$ when $N(w)$ is a neighborhood of $w$. Now there exists a balanced neighborhood $N$ of 0 such that $N+w \subseteq N(w)$, and there is a $y \in N$ such that $u(y)$ is rational and $u(y) \neq 1$. Then $w+y, w-y \in N(w)$, so by (2.2) 


$$
2 c=u(w+y)+u(w-y)=2 u(w) u(y)=2 c u(y) .
$$

Hence $c=0$, which is a contradiction.

Now suppose $u(y)$ is irrational and $N(y)$ is a neighborhood of $y$. Since $u$ is nonconstant on $N(y)$, as before, by the intermediate value theorem, there is a $w \in N(y)$ such that $u(w)$ is rational. In this way we obtain a net $w_{\alpha}$ such that $w_{\alpha} \rightarrow y$ and $u\left(w_{\alpha}\right)$ are rational. Since $u$ is continuous, by (2.2) we have for every $x \in V$

$$
\begin{aligned}
u(x+y)+u(x-y) & =\lim \left[u\left(x+w_{\alpha}\right)+u\left(x-w_{\alpha}\right)\right] \\
& =\lim 2 u(x) u\left(w_{\alpha}\right)=2 u(x) u(y) .
\end{aligned}
$$

We conclude that (2.2) holds for every $x, y \in V$ and, in particular, (2.3) holds for every $y \in V$. Letting $x=0$ in (2.2) gives $u(-y)=u(y)$ for all $y \in V$, so $u$ is even.

Define $g: V \rightarrow \mathbb{C}$ by $g(x)=u(x)+i u(x+z)$ where $z$ is a zero of $u$. Then for every $x, y \in V$, applying (2.2), (2.1) and evenness gives

$$
\begin{aligned}
g(x) g(y) & =u(x) u(y)-u(x+z) u(y+z)+i[u(y) u(x+z)+u(x) u(y+z)] \\
& =u(x+y)+i u(x+y+z)=g(x+y) .
\end{aligned}
$$

Moreover, by (2.3) we have

$$
|g(x)|^{2}=[u(x)]^{2}+[u(x+z)]^{2}=1 .
$$

Hence, $g$ is a continuous character on $V$. It follows from a result in [11] that there exists a continuous linear functional $f: V \rightarrow \mathbb{R}$ such that $g(x)=e^{i f(x)}$ for all $x \in V$. Hence $u(x)=\operatorname{Re} g(x)=\cos f(x)$. In the general case, when $u(0) \neq 1$ we obtain $u(x)=u(0) \cos f(x)$.

For the second case, $u$ has no zero. Suppose there is a $w \in V$ such that $u(w)<0$. Now there exists a balance neighborhood $N$ of 0 such that $w \in N$. Since $u(0)=1$, by the intermediate value theorem there is a $y \in N$ such that $u(y)=0$. This gives a contradiction, so $u(w)>0$ for all $w \in V$. Now assume that $u(y)=s / t$ is rational, where $s, t$ are positive integers. Let $x_{1}=\cdots=x_{s}=0, y_{1}=\cdots=y_{t}=y$. Then

$$
\sum_{i=1}^{t} u\left(y_{i}\right)=t u(y)=s u(0)=\sum_{i=1}^{s} u\left(x_{i}\right) \text {. }
$$

Since $u$ is C2, applying (1.3) gives for every $x \in V, t[u(x+y)+u(x-y)]$ $=2 s u(x)$. Hence (2.2) again holds whenever $u(y)$ is rational. By the same argument used before, we conclude that (2.2) and (2.3) hold for every $x, y \in$ $V$.

We now show that $u(x) \geq 1$ for all $x \in V$. Let $a_{1}=2^{-1 / 2}$, and for any integer $n>1$ let $a_{n}=\left[\left(a_{n-1}+1\right) / 2\right]^{1 / 2}$. We prove by induction on $n$ that $u(x) \geq a_{n}$ for all $x \in V$. Applying (2.3) we have

$$
[u(x)]^{2}+\frac{1}{2}+\frac{1}{2} u(2 x) \geq \frac{1}{2} .
$$

Hence $u(x) \geq a_{1}$ for all $x \in V$, so the result holds for $n=1$. Suppose the result holds for the integer $n \geq 1$. Applying (2.3) gives

$$
[u(x)]^{2}=\frac{1}{2}+\frac{1}{2} u(2 x) \geq \frac{1}{2}\left(1+a_{n}\right),
$$


so $u(x) \geq a_{n+1}$ for all $x \in V$, which completes the induction proof. We next show that $a_{n} \leq 1$ for all $n$. Indeed, $a_{1} \leq 1$ and if $a_{n} \leq 1$ then $a_{n+1}^{2}=$ $\left(a_{n}+1\right) 2 \leq 1$, so the result follows by induction. Finally, $a_{n}$ is an increasing sequence since

$$
a_{n}=\left[\frac{1}{2}\left(a_{n-1}+1\right)\right]^{1 / 2} \geq\left[\frac{1}{2}\left(a_{n-1}+a_{n-1}\right)\right]^{1 / 2}=\left(a_{n-1}\right)^{1 / 2} \geq a_{n-1} .
$$

Letting $L=\lim a_{n}$, it follows that $L^{2}=(L+1) / 2$. Solving this equation gives $L=1$. We conclude that $u(x) \geq L=1$ for all $x \in V$.

Since $u \neq 1$, there exists a $w \in V$ such that $u(w)>1$. Let $c=$ $[2(u(w)-1)]^{-1 / 2}$ and define $g: V \rightarrow \mathbb{R}$ by

$$
g(x)=u(x)+c[u(x+w / 2)-u(x-w / 2)] .
$$

Then a straightforward calculation (see [4, p. 220]) gives $g(x+y)=g(x) g(y)$ for all $x, y \in V$. It follows that $g(2 x)=[g(x)]^{2} \geq 0$ so $g(x) \geq 0$ for all $x \in V$. In fact, $g(x)>0$ for all $x \in V$. Indeed, if $g(y)=0$ then $g(x+y)=0$ for all $x \in V$. In particular, $g(0)=0$ but by $(2.4), g(0)=1$, which is a contradiction. Letting $f(x)=\log g(x)$, we have $f(x+y)=f(x)+f(y)$ for all $x, y \in V$. Since $f$ is continuous, we conclude that $f$ is a continuous linear functional on $V$. From (2.4) we have

$$
g(-x)=u(x)-c[u(x+w / 2)-u(x-w / 2)] .
$$

Hence

$$
u(x)=\frac{g(x)+g(-x)}{2}=\frac{e^{f(x)}+e^{-f(x)}}{2}=\cosh f(x) .
$$

Again, in the general case, we have $u(x)=u(0) \cosh f(x)$.

For uniqueness, suppose $u \neq 0$. If $u=1$ then $f=0$ is the unique solution, so suppose $u \neq 1$. Clearly, if $g: V \rightarrow \mathbb{R}$ is a continuous linear functional, we cannot have

$$
u(x)=u(0) \cos f(x)=u(0) \cosh g(x),
$$

so suppose

$$
u(x)=u(0) \cos f(x)=u(0) \cos g(x)
$$

for all $x \in V$. Now there exists a neighborhood $N$ of 0 such that $|f(x)|$, $|g(x)|<\pi / 2$ for all $x \in N+N$ [13]. It follows from (2.5) that if $w \in N+N$ then $g(w)= \pm f(w)$. Now suppose $x, y \in N$ and $g(x)=f(x) \neq 0$ while $g(y)=-f(y) \neq 0$. Then

$$
g(x+y)=g(x)+g(y)=f(x)-f(y) .
$$

Now suppose $g(x+y)=f(x+y)$. Then $f(x)+f(y)=f(x)-f(y)$, which gives the contradiction $f(y)=0$. Similarly, if $g(x+y)=-f(x+y)$, we obtain the contradiction $f(x)=0$. Hence either $g(x)=f(x)$ for $g(x)=-f(x)$ for all $x \in N$. Suppose the first case holds and $w \in V$. Since $N$ is absorbing, there is a $t \in \mathbb{R} \backslash\{0\}$ such that $t w \in N$. Hence $\operatorname{tg}(w)=g(t w)=f(t w)=t f(w)$, so $g=f$. Similarly, in the second case, $g=-f$. The proof is similar for the case

$$
u(x)=u(0) \cosh f(x)=u(0) \cosh g(x) \text {. }
$$


Corollary 2. If $u: V \rightarrow \mathbb{R}$ is continuous, $\mathrm{C} 1$, and has a zero, then there exists a continuous linear functional $f: V \rightarrow \mathbb{R}$ such that $u(x)=u(0) \cos f(x)$.

Proof. This follows from the first half of the proof of Theorem 1.

Corollary 3. Let $V$ be a real Hilbert space with inner product $\langle\cdot, \cdot\rangle$. If $u: V \rightarrow$ $\mathbb{R}$ is continuous and $\mathrm{C} 2$, then there exists a $y \in V$ such that $u(x)=u(0) \cos \langle x, y\rangle$ or $u(x)=u(0) \cosh \langle x, y\rangle$ for all $x \in V$. Moreover, $y$ is unique in the sense that if $u \neq 0$ and either $u(x)=u(0) \cos \langle x, z\rangle$ or $u(x)=u(0) \cosh \langle x, z\rangle$ for all $x \in V$, then $z= \pm y$.

Proof. This follows from Theorem 1 since every continuous linear functional on $V$ has the form $f(x)=\langle x, y\rangle$ for some $y \in V$.

Corollary 4. Let $V$ be a real Hilbert space with inner product $\langle\cdot, \cdot\rangle$. If $u: V \rightarrow$ $\mathbb{R}$ is continuous, $\mathrm{C} 1$, and has a zero, then there exists a $y \in V$ such that $u(x)=u(0) \cos \langle x, y\rangle$. If $u \neq 0$ then the elements $\pm \pi y / 2\|y\|^{2}$ are the unique zeros of $u$ with smallest norm.

Proof. We only need to prove the last statement. Notice that $y \neq 0$ since $u$ has a zero. Letting $z=\pi y / 2\|y\|^{2}$, we have

$$
u( \pm z)=u(0) \cos ( \pm \pi / 2)=0 \text {, }
$$

so $\pm z$ are zeros of $u$. Let $w$ be an arbitrary zero of $u$. Then $0=u(w)=$ $u(0) \cos \langle w, y\rangle$. It follows that $\langle w, y\rangle=n \pi / 2$ where $n$ is an odd integer. By Schwarz's inequality

$$
|n| \pi / 2=|\langle w, y\rangle| \leq\|w\|\|y\| \text {. }
$$

Hence

$$
\|w\| \geq|n| \pi / 2\|y\|=|n|\|z\| \geq\|z\|,
$$

so $\pm z$ are zeros with minimal norm. If $w$ is a zero of $u$ with $\|w\|=\|z\|$, then by (2.6), $n= \pm 1$ so $|\langle w, y\rangle|=\pi / 2$. Hence

$$
|\langle w, z\rangle|=\frac{\pi}{2\|y\|^{2}}|\langle w, y\rangle|=\left(\frac{\pi}{2\|y\|}\right)^{2}=\|w\|\|z\| .
$$

Since we have equality in Schwarz's inequality, it follows that $w= \pm z$.

We close with some remarks concerning the proof of Theorem 1 . In the first half of the proof, we showed that $u$ was $\mathrm{Cl}$. Although this was not necessary for the proof of Theorem 1 , it is necessary for Corollary 2 .

The bulk of the proof was to show that a continuous C2 (or C1) function satisfies d'Alembert's equation. After that, the proof was fairly standard. One could proceed differently at this point by employing the following theorem $[4$, $12,14]$.

Theorem 5. The general complex-valued solutions of d'Alembert's functional equation on the cartesian square of an abelian group $G$ are given by

$$
u(x)=(h(x)+h(-x)) / 2
$$

where $h$ satisfies $h(x+y)=h(x) h(y)$ for all $x, y \in G$.

In our case, $u$ is continuous and it follows that $h$ is also continuous. One can now argue that there exists a continuous linear functional $f: V \rightarrow \mathbb{C}$ such 
that $h(x)=e^{f(x)}$ for all $x \in V$. It follows that $u(x)=\cosh f(x)$ for all $x \in V$. Letting $f_{1}$ and $f_{2}$ be the real and imaginary parts of $f$, respectively, we then have

$$
\begin{aligned}
u(x) & =\cosh \left[f_{1}(x)+i f_{2}(x)\right] \\
& =\cosh f_{1}(x) \cos f_{2}(x)-i \sinh f_{1}(x) \sin f_{2}(x) .
\end{aligned}
$$

Since $u(x)$ is real, one can further argue that either $f_{1}=0$ or $f_{2}=0$. The amount of work required in providing the details for this method is about the same as that employed in the present proof.

\section{REFERENCES}

1. J. Aczél, Lectures on functional equations and their applications, Academic Press, New York, 1966.

2. __ On applications and theory of functional equations, Academic Press, New York, 1969.

3. J. Aczél, J. Chung, and C. Ng, Symmetric second differences in product form on groups, Topics in Mathematical Analysis, World Scientific Publ., Singapore, 1989.

4. J. Aczél and J. Dhombres, Functional equations in several variables, Encyclopedia Math. Appl., vol., Cambridge Univ. Press, Cambridge, England, 1989.

5. J. d'Alembert, Addition au Mémoire sur la courbe que forme une corde tendue mise en vibration, Hist. Acad. Berlin (1750), 355-360.

6. __ Mémoire sur les principles de mécanique, Hist. Acad. Sci. Paris (1769), 278-286.

7. J. A. Baker, The stability of the cosine equation, Proc. Amer. Math. Soc. 80, (1980), 411416.

8. G. Hemion, A discrete geometry: speculations on a new framework for classical electrodynamics, Intern. J. Theor. Phys. 27 (1988), 1145-1255.

9. __ Quantum mechanics in a discrete model of classical physics, Intern. J. Theor. Phys. 29 (1990), 1335-1368.

10. E. Hewitt and K. Ross, Abstract harmonic analysis vol. I, Academic Press, New York, 1963.

11. E. Hewitt and H. S. Zuckerman, A group-theoretic method in approximation theory, Ann. of Math. (2) 52 (1950), 557-567.

12. Pl. Kannappan, The functional equation $f(x y)+f\left(x y^{-1}\right)=2 f(x) f(y)$ for groups, Proc. Amer. Math. Soc. 19 (1968), 69-74.

13. A. Wilansky, Modern methods in topological vector spaces, McGraw-Hill, New York, 1978.

14. W. Wilson, On certain related functional equations, Bull. Amer. Math. Soc. 26 (1919), 300312.

Department of Mathematics and Computer Science, University of Denver, Denver, COLORADO 80208 